\title{
SMOOTH POTENTIALS WITH PRESCRIBED BOUNDARY BEHAVIOUR
}

\author{
Stephen J. Gardiner and Anders Gustafsson
}

\begin{abstract}
This paper examines when it is possible to find a smooth potential on a $C^{1}$ domain $D$ with prescribed normal derivatives at the boundary. It is shown that this is always possible when $D$ is a Liapunov-Dini domain, and this restriction on $D$ is essential. An application concerning $C^{1}$ superharmonic extension is given.
\end{abstract}

\section{Results}

Let $D$ be a $C^{1}$ domain in Euclidean space $\mathbb{R}^{n}$, where $n \geq 2$. Thus $D$ is bounded, $\partial D$ can be represented locally as the graph of a $C^{1}$ function of $n-1$ variables, and there is a uniquely defined inward normal $n_{z}$ at each point $z$ of $\partial D$. We denote by $C^{1}(\bar{D})$ the collection of continuous functions on $\bar{D}$ which possess a continuous gradient on $D$ that extends continuously to $\bar{D}$.

This paper is concerned with whether it is possible to find a smooth potential on $D$ with prescribed normal derivatives on the boundary. More precisely, given a continuous function $g: \partial D \rightarrow(0,+\infty)$, we ask if there is a function $v \in C^{1}(\bar{D})$ which is superharmonic on $D$ and satisfies the boundary conditions

$$
v(z)=0 \quad \text { and } \quad \frac{\partial v}{\partial n_{z}}=g(z) \quad(z \in \partial D),
$$

where $\partial / \partial n_{z}$ denotes differentiation in the direction of the inward normal at $z$. The answer will be given in Theorem 1 below.

By a Dini function we mean an increasing continuous function $\varepsilon:(0,+\infty) \rightarrow(0,+\infty)$ such that $\varepsilon(t) / t^{\gamma}$ is decreasing on $(0,1)$ for some $\gamma \in(0,1)$ and

$$
\int_{0}^{1} \frac{\varepsilon(t)}{t} d t<+\infty
$$

2000 Mathematics Subject Classification. 31B15.

Key words. Potential, boundary behaviour, superharmonic extension. 
A $C^{1}$ domain $D$ is called a Liapunov-Dini domain (cf. [11]) if there is a Dini function $\varepsilon$ such that the angle between the normals $n_{y}$ and $n_{z}$ at any two points $y, z \in \partial D$ does not exceed $\varepsilon(\|y-z\|)$. Examples include the $C^{1, \alpha}$-domains $(0<\alpha<1)$, which correspond to the case where $\varepsilon(t)=t^{\alpha}$.

Theorem 1. Let D be a Liapunov-Dini domain. Then, for each continuous function $g: \partial D \rightarrow(0,+\infty)$, there is a function $v \in C^{1}(\bar{D})$ which is superharmonic on $D$ and satisfies (1).

The function $v$ of Theorem 1 is certainly not unique: as will be clear from the proof it can be chosen to be harmonic on any predetermined open subset $U$ of $D$ which satisfies $\bar{U} \subset D$. We remark that Theorem 1 is related to work of Wallin [10] on the extension, in the form of potentials, of continuous functions from compact polar sets.

The example below shows the relevance of condition (2) to Theorem 1.

Example 1. Let $\varepsilon:[0,+\infty) \rightarrow[0,+\infty)$ be an increasing continuous function such that $\varepsilon(0)=0$ and (2) fails to hold. (For example, we could choose $\varepsilon(t)=\left\{1+\log ^{+}(e / t)\right\}^{-1}$.) Further, let $D$ be a $C^{1}$ domain such that

$$
D \cap\{\|x\|<1\}=\left\{\left(x^{\prime}, x_{n}\right) \in \mathbb{R}^{n-1} \times \mathbb{R}: x_{n}>-\psi\left(\left\|x^{\prime}\right\|\right)\right\} \cap\{\|x\|<1\}
$$

where $\psi(t)=\int_{0}^{t} \varepsilon(s) d s$. Then the only function $v$ in $C(\bar{D})$ which is superharmonic on $D$, valued 0 on $\partial D$ and has a finite normal derivative at 0 , is the zero function.

We give below an application of Theorem 1 to superharmonic extension.

Corollary 1. Let D be a Liapunov-Dini domain (with Dini function $\varepsilon$ ) such that $\mathbb{R}^{n} \backslash \bar{D}$ is connected. Suppose that $u \in C^{1}(\bar{D})$, where $\left.u\right|_{D}$ is superharmonic and, for each $z \in \partial D$, there is a linear polynomial $L_{z}$ such that

$$
\left|u(x)-L_{z}(x)\right| \leq \varepsilon(\|x-z\|)\|x-z\| \quad(x \in \partial D) .
$$

Then there is a superharmonic function $\bar{u} \in C^{1}\left(\mathbb{R}^{n}\right)$ such that $\bar{u}=u$ on $\bar{D}$.

Corollary 1 is related to a question raised by Verdera, Mel'nikov and Paramonov [9] concerning $C^{1}$ extension of superharmonic functions. We do not know if condition (3) can be omitted.

We will establish Theorem 1, Example 1 and Corollary 1 in Sections 3-5 respectively, following some preliminary material in Section 2. 
Acknowledgement. This work was supported by EU Research Training Network Contract HPRN-CT-2000-00116.

\section{Preliminaries}

We write $C_{a}$ for a positive constant, depending at most on $a$, not necessarily the same on any two occurrences, and assume, without loss of generality, that $0 \in D$. We write $\delta(x)$ for the distance of a point $x$ from $\partial D$, denote the Green function for $D$ by $G_{D}(\cdot, \cdot)$, and define

$$
M(z, y)=\lim _{x \rightarrow z, x \in D} \frac{G_{D}(x, y)}{G_{D}(x, 0)} \quad(z \in \partial D ; y \in D) .
$$

(This is the "Martin kernel" for $D$; see [2, Chapter 8].)

Lemma A. Let $D$ be a Liapunov-Dini domain. Then:

(i) $G_{D}(x, 0) \leq C_{D} \delta(x)\|x\|^{1-n} \quad(x \in D)$;

(ii) $G_{D}(\cdot, y) \in C^{1}(\bar{D} \backslash\{y\}) \quad(y \in D)$;

(iii) for each $y \in D$ the function $z \mapsto \frac{\partial}{\partial n_{z}} G_{D}(\cdot, y)$ is positive and continuous on $\partial D$;

(iv) $M\left(x^{*}, x\right) \geq C_{D}\{\delta(x)\}^{1-n} \quad(x \in D)$, where $x^{*}$ is any point of $\partial D$ satisfying $\left\|x-x^{*}\right\|=\delta(x)$.

When $n \geq 3$ assertions (i)-(iii) above may be found in Theorems 2.32.5 of $[\mathbf{1 1}]$, and (iv) follows from an estimate on p. 28 of that paper. In two dimensions the lemma can be verified using a conformal mapping argument, even under somewhat weaker hypotheses on $D$ (cf. [ 7 , Theorem 3.5] for the case where $D$ is simply connected).

The next result is a special case of Theorem 1 of $[\mathbf{1}]$. As usual, $B(x, r)$ denotes the open ball of centre $x$ and radius $r$ in $\mathbb{R}^{n}$.

Lemma B (Boundary Harnack Principle). There are constants $R>0$, $a_{0}>1$ and $c_{0}>1$, depending only on $D$, with the following property: if $z \in \partial D$ and $0<r<R$, and $h_{1}, h_{2}$ are positive harmonic functions on $D \cap B\left(z, a_{0} r\right)$ that vanish continuously on $\partial D \cap B\left(z, a_{0} r\right)$, then

$$
\frac{h_{1}(x)}{h_{2}(x)} \leq c_{0} \frac{h_{1}(y)}{h_{2}(y)} \quad(x, y \in D \cap \overline{B(z, r)}) .
$$

\section{Proof of Theorem 1}

For each $y \in D$ let $B_{y}=B(y, \delta(y) / 2)$, and let

$$
D(r)=\{x \in D: \delta(x)<r\} \quad(r>0) .
$$


We note that

$$
\|x-y\| \geq \frac{\delta(x)}{3} \quad\left(x \in D \backslash B_{y}\right),
$$

for otherwise there exists $x \in D \backslash B_{y}$ such that

$$
\|z-x\|-\|z-y\|<\frac{\delta(x)}{3} \quad\left(z \in \mathbb{R}^{n}\right)
$$

whence

$$
\|z-y\|>\frac{2 \delta(x)}{3} \quad(z \in \partial D)
$$

and we obtain the contradictory conclusion that

$$
\delta(y) \geq \frac{2 \delta(x)}{3}>2\|x-y\| .
$$

Now let $R, a_{0}$ and $c_{0}$ be as in Lemma B (we choose $R$ small enough so that $2 a_{0} R<\delta(0)$ ), and let $y \in D(R / 2)$. We claim that

$$
\frac{G_{D}(x, y)}{G_{D}(x, 0)} \leq C_{D} M\left(x^{*}, y\right) \quad\left(x \in D(R) \backslash B_{y}\right)
$$

where $x^{*}$ denotes any point of $\partial D$ satisfying $\left\|x-x^{*}\right\|=\delta(x)$. To see this we define

$$
\rho=\min \left\{\delta(x), \frac{\left\|x^{*}-y\right\|}{a_{0}}\right\},
$$

whence $\rho<R$. The choice of $\rho$ and $R$ ensure that the functions $G_{D}(\cdot, y)$ and $G_{D}(\cdot, 0)$ are harmonic on $D \cap B\left(x^{*}, a_{0} \rho\right)$, so we can apply the boundary Harnack principle to see that

$$
\frac{G_{D}(z, y)}{G_{D}(z, 0)} \leq c_{0} M\left(x^{*}, y\right) \quad\left(z \in D \cap \overline{B\left(x^{*}, \rho\right)}\right) .
$$

If $\delta(x) \leq\left\|x^{*}-y\right\| / a_{0}$, then $\rho=\delta(x)$ and the inequality in (5) clearly holds. It remains to consider the case of (5) where $\delta(x)>\left\|x^{*}-y\right\| / a_{0}$, and so

$$
\|x-y\|>\frac{\left\|x^{*}-y\right\|}{3 a_{0}}=\frac{\rho}{3}
$$

by (4). Let

$$
z_{1} \in D \cap B\left(x^{*}, \rho\right) \quad \text { and } \quad z_{2} \in D \cap \partial B(y, \rho / 3) .
$$

Then

$$
\left\|z_{1}-z_{2}\right\| \leq\left\|z_{1}-x^{*}\right\|+\left\|x^{*}-y\right\|+\left\|y-z_{2}\right\| \leq\left(a_{0}+4 / 3\right) \rho
$$

and

$$
\left\|z_{1}-y\right\| \geq\left\|y-x^{*}\right\|-\left\|z_{1}-x^{*}\right\|>\left(a_{0}-1\right) \rho
$$


whence $z_{1} \notin B(y, \rho / 3)$ provided we arrange that $a_{0}>4 / 3$. We note that $\delta\left(z_{1}\right), \delta\left(z_{2}\right) \in\left(0, \rho\left(a_{0}+1 / 3\right)\right]$, so $z_{1}, z_{2} \notin B(0, R)$ in view of our choice of $R$. Since $D$ is $C^{1}$, we can join $z_{1}$ to $z_{2}$ by a curve $\gamma$ in $D \backslash[B(y, \rho / 3) \cup$ $B(0, R)$ ], of length at most $C_{D} \rho$. Further, we can choose $c_{1}>0$, depending only on $D$, such that, for each $z \in \gamma$, either

$$
B\left(z, 2 c_{1} \rho\right) \subset D \backslash\{0, y\}
$$

or

$$
B\left(z, c_{1} \rho\right) \subset B\left(z^{*}, 3 c_{1} \rho\right) \quad \text { and } \quad 0, y \notin B\left(z^{*}, 3 a_{0} c_{1} \rho\right) .
$$

Thus (6), together with repeated use of Harnack's inequalities and the boundary Harnack principle as appropriate, yields

$$
\frac{G_{D}\left(z_{2}, y\right)}{G_{D}\left(z_{2}, 0\right)} \leq C_{D} M\left(x^{*}, y\right) \quad\left(z_{2} \in D \cap \partial B(y, \rho / 3)\right),
$$

and it follows from the minimum principle that

$$
C_{D} M\left(x^{*}, y\right) G_{D}(\cdot, 0)-G_{D}(\cdot, y)>0 \quad \text { on } \quad D \backslash B(y, \rho / 3) .
$$

The claim (5) now holds in view of (7).

Using (4) and a well known consequence of Harnack's inequalities (see [2, Corollary 1.4.2]), we observe that

$$
\left\|\nabla_{x} G_{D}(x, y)\right\| \leq \frac{3 n}{\delta(x)} G_{D}(x, y) \quad\left(x \in D \backslash B_{y}\right),
$$

and hence

$$
\left\|\nabla_{x} G_{D}(x, y)\right\| \leq C_{D} \frac{G_{D}(x, y)}{G_{D}(x, 0)} \quad\left(x \in D(R) \backslash B_{y}\right),
$$

by Lemma A(i). Now let $v_{y}$ denote the (Green) potential on $D$ of normalized Lebesgue measure on $B_{y}$. By the mean value property of harmonic functions, (9) and then (5) we see that

(10) $\left\|\nabla_{x} v_{y}(x)\right\|=\left\|\nabla_{x} G_{D}(x, y)\right\| \leq C_{D} M\left(x^{*}, y\right) \quad\left(x \in D(R) \backslash B_{y}\right)$.

Further, if we define $v_{y}=0$ on $\partial D$, then it follows from Lemma A(ii) (and [2, Theorem 4.5.3]) that $v_{y} \in C^{1}(\bar{D})$, and

$$
\left\|\nabla_{x} v_{y}(x)\right\| \leq C_{n}\{\delta(y)\}^{1-n} \quad\left(x \in \partial B_{y}\right)
$$

in view of (8). Since $\Delta v_{y}=-C_{n}\{\delta(y)\}^{-n}$ on $B_{y}$, the components of $\nabla_{x} v_{y}$ are harmonic there, and so

$$
\left\|\nabla_{x} v_{y}(x)\right\| \leq C_{n}\{\delta(y)\}^{1-n} \quad\left(x \in B_{y}\right) .
$$

From Lemma A(iv) and Harnack's inequalities we see that

$$
\left\|\nabla_{x} v_{y}(x)\right\| \leq C_{D} M\left(x^{*}, x\right) \leq C_{D} M\left(x^{*}, y\right) \quad\left(x \in B_{y}\right) .
$$


Combining this with (10) we obtain

$$
\left\|\nabla_{x} v_{y}(x)\right\| \leq C_{D} M\left(x^{*}, y\right) \quad(x \in D(R)),
$$

whence

$$
v_{y}(x) \leq C_{D} \delta(x) M\left(x^{*}, y\right) \quad(x \in D(R)) .
$$

Now let $g: \partial D \rightarrow(0,+\infty)$ be continuous. By Lemma A(iii) and a special case of Theorem 3 in [6] (cf. [3, Theorem 10]), there are sequences $\left(y_{k}\right)$ in $D(R / 2)$ and $\left(a_{k}\right)$ in $[0,+\infty)$ such that

$$
\frac{g(z)}{\frac{\partial}{\partial n_{z}} G_{D}(\cdot, 0)}=\sum_{k=1}^{\infty} a_{k} M\left(z, y_{k}\right) \quad(z \in \partial D),
$$

and the convergence is uniform on $\partial D$ in view of Dini's theorem. It follows from (12) that the series $\sum a_{k} v_{y_{k}}$ converges (uniformly) on $\overline{D(R)}$, and hence on $\bar{D}$ by the maximum principle (each $v_{y_{k}}$ is harmonic on $D \backslash \overline{D(R)}$ ). We denote the sum of this series by $v$. By (11) the series $\sum a_{k}\left\|\nabla v_{y_{k}}\right\|$ also converges uniformly on $\bar{D}$. It follows that $v \in$ $C^{1}(\bar{D})$ and that

$$
\begin{aligned}
\frac{\partial v}{\partial n_{z}} & =\sum a_{k} \frac{\partial}{\partial n_{z}} v_{y_{k}} \\
& =\sum a_{k} \frac{\partial}{\partial n_{z}} G_{D}\left(\cdot, y_{k}\right) \\
& =\left\{\sum a_{k} M\left(z, y_{k}\right)\right\} \frac{\partial}{\partial n_{z}} G_{D}(\cdot, 0) \\
& =g(z) \quad \text { when } z \in \partial D,
\end{aligned}
$$

by (13). Thus (1) holds, since clearly $v=0$ on $\partial D$ in view of (12). Finally, each $v_{y_{k}}$ is superharmonic on $D$, so the same is true of $v$. Theorem 1 is now proved.

\section{Details of Example 1}

Let $D$ be as stated in Example 1 and let $z_{t}=(0, \ldots, 0, t)$. The failure of (2) to hold implies that

$$
\frac{G_{D}\left(z_{t}, z_{1 / 2}\right)}{t} \rightarrow+\infty \quad(t \rightarrow 0+)
$$

(see [4, Corollary 4.3]; cf. [8, p. 377] when $n=2$ ). Now suppose that $v \in C(\bar{D})$ and that $v$ is superharmonic on $D$ and valued 0 on $\partial D$. By the Riesz decomposition theorem $v$ is of the form $v(x)=\int G_{D}(x, \cdot) d \mu$ 
on $D$ for some measure $\mu$. If $\mu \neq 0$, then Harnack's inequalities, applied to $G_{D}(x, \cdot)$, show that there are positive constants $a, c$ such that

$$
v\left(z_{t}\right) \geq c G_{D}\left(z_{t}, z_{1 / 2}\right) \quad(0<t<a),
$$

and it follows from (14) that $v$ does not have a finite normal derivative at 0 .

\section{Proof of Corollary 1}

Let $D$ and $u$ be as in the statement of Corollary 1 , and let $\Omega=\mathbb{R}^{n} \backslash \bar{D}$. By hypothesis $\Omega$ is connected. In view of condition (3) and $[\mathbf{1 1}$, Theorem 2.4], the solution $w$ to the Dirichlet problem in $\Omega$, with boundary data $u$ on $\partial D$ and 0 at $\infty$, satisfies $w \in C^{1}(\bar{\Omega})$, where $w=u$ on $\partial D$.

If $n \geq 3$, then we define $h_{0}$ to be the harmonic measure of $\{\infty\}$ in $\Omega$; if $n=2$, then we define $h_{0}$ to be the Green function for $\Omega \cup\{\infty\}$ with pole at $\infty$. In either case we define $h_{0}=0$ on $\partial D$ and note from Lemma $A$ and the Kelvin transform that $h_{0} \in C^{1}(\bar{\Omega})$ and $-\partial h_{0} / \partial n_{z}$ is a positive continuous function of $z$ in $\partial D$. (We always use $n_{z}$ to denote the inward normal at $z$ relative to $D$.)

We now choose $a>0$ large enough so that the continuous function

$$
g(z)=\frac{\partial w}{\partial n_{z}}-\frac{\partial u}{\partial n_{z}}-a \frac{\partial h_{0}}{\partial n_{z}} \quad(z \in \partial D)
$$

is positive on $\partial D$. By Theorem 1 and inversion there is a function $v \in$ $C^{1}(\bar{\Omega})$ such that $\left.v\right|_{\Omega}$ is superharmonic on $\Omega$ and

$$
v(z)=0 \quad \text { and } \quad-\frac{\partial v}{\partial n_{z}}=g(z) \quad(z \in \partial D) .
$$

For each $b \geq a$, let

$$
u_{b}(x)=\left\{\begin{array}{ll}
u(x) & (x \in \bar{D}) \\
w(x)+v(x)-b h_{0}(x) & (x \in \Omega)
\end{array} .\right.
$$

Since $v-b h_{0}=0$ on $\partial D$, the functions $u_{b}$ are continuous on $\mathbb{R}^{n}$. Further, by (16) and then (15),

$$
\frac{\partial}{\partial n_{z}}\left(w+v-a h_{0}\right)=\frac{\partial w}{\partial n_{z}}-g(z)-a \frac{\partial h_{0}}{\partial n_{z}}=\frac{\partial u}{\partial n_{z}} \quad(z \in \partial D),
$$

so $u_{a} \in C^{1}\left(\mathbb{R}^{n}\right)$. It remains to establish the superharmonicity of $u_{a}$. Clearly, it will be enough to check the superharmonicity of $u_{b}$ when $b>a$, and then let $b \rightarrow a+$. Further, since we know that $u_{b}$ is superharmonic both on $D$ and on $\Omega$, we need only verify the superharmonic mean value inequality at points of $\partial D$. 
We will do this using an argument of Carroll [5], which we include here for the sake of completeness. Let $z \in \partial D$ and $r>0$, and let $h$ be the harmonic extension of $u_{b}$ from $\partial B(z, r)$ to $\overline{B(z, r)}$. Further, let $c$ denote the minimum value of $u_{b}-h$ on $\overline{B(z, r)}$, and suppose, for the sake of contradiction, that $c<0$. Then the value $c$ is attained by $u_{b}-h$ at some point $y \in B(z, r)$. The minimum principle, applied on $B(z, r) \backslash \partial D$, shows that $y \in \partial D \cap B(z, r)$. By considering $u_{b}-h$ separately on $\bar{D}$ and on $\bar{\Omega}$, we obtain

$$
\frac{\partial}{\partial n_{y}}\left(u_{a}-h\right) \geq 0 \geq \frac{\partial}{\partial n_{y}}\left(u_{a}-h\right)-(b-a) \frac{\partial h_{0}}{\partial n_{y}},
$$

which contradicts the fact that $\partial h_{0} / \partial n_{y}<0$. Thus $c=0$, and $u_{b} \geq h$ on $B(z, r)$, whence $u_{b}(z) \geq h(z)$, as required. Corollary 1 is now established.

\section{References}

[1] H. Aikawa, Boundary Harnack principle and Martin boundary for a uniform domain, J. Math. Soc. Japan 53(1) (2001), 119-145.

[2] D. H. Armitage and S. J. Gardiner, "Classical potential theory", Springer Monographs in Mathematics, Springer-Verlag, London, 2001.

[3] F. F. Bonsall and D. Walsh, Vanishing $l^{1}$-sums of the Poisson kernel, and sums with positive coefficients, Proc. Edinburgh Math. Soc. (2) 32(3) (1989), 431-447.

[4] K. BuRdzy, Brownian excursions and minimal thinness. II. Applications to boundary behavior of the Green function, in: "Seminar on stochastic processes, 1985" (Gainesville, Fla., 1985), Progr. Probab. Statist. 12, Birkhäuser, Boston, MA, 1986, pp. 35-62.

[5] T. F. CARroll, A classical proof of Burdzy's theorem on the angular derivative, J. London Math. Soc. (2) 38(3) (1988), 423-441.

[6] S. J. Gardiner and J. PAU, Approximation on the boundary and sets of determination for harmonic functions, Illinois J. Math. 47(4) (2003), 1115-1136.

[7] Ch. Pommerenke, "Boundary behaviour of conformal maps", Grundlehren der Mathematischen Wissenschaften 299, SpringerVerlag, Berlin, 1992.

[8] M. Tsusi, "Potential theory in modern function theory", Reprinting of the 1959 original, Chelsea Publishing Co., New York, 1975.

[9] J. Verdera, M. S. Mel'nikov and P. V. Paramonov, $C^{1}$-approximation and the extension of subharmonic functions, (Russian), 
Mat. Sb. 192(4) (2001), 37-58; translation in: Sb. Math. 192(3-4) (2001), 515-535.

[10] H. WALLin, Continuous functions and potential theory, Ark. Mat. 5 (1963), 55-84.

[11] K.-O. Widman, Inequalities for the Green function and boundary continuity of the gradient of solutions of elliptic differential equations, Math. Scand. 21 (1967), 17-37.

Stephen J. Gardiner:

Department of Mathematics

University College Dublin

Dublin 4

Ireland

E-mail address: stephen.gardiner@ucd.ie

Anders Gustafsson:

Current address:

Institutionen för kemi och biomedicinsk vetenskap

Högskolan i Kalmar

39182 Kalmar

Sweden

E-mail address: anders.gustafsson@math.umu.se

Rebut el 18 de juliol de 2003. 
